\title{
A STUDY OF RINGS WITH TRIVIAL PRERADICAL IDEALS
}

\author{
(Dedicated to Professor Goro Azumaya for the celebration
} of his sixtieth birthday)

By

Hisao KATAYAMA

\section{Introduction.}

Our purpose is to study those rings without non-trivial preradical ideals of idempotent preradicals (or exact radicals), supplying the cases of idempotent radicals by [2], of left exact preradicals by $[1,6,14,17]$ and of left exact radicals by $[2,6]$. In Theorem 3.1 , we shall show that a ring $R$ has no nontrivial idempotent preradical ideals if and only if every nonzero left ideal is a generator for $R$-mod (left $G$-ring). Generalizing this, we consider those rings whose nonzero finitely generated (or cyclic, essential) left ideals are generators. We shall give several examples which distinguish those rings to be refered.

\section{Preliminaries.}

This section consists of a list of definitions and properties of some type of preradicals treated in this paper. In particular, we shall give the bijections of those preradicals for Morita equivalent rings.

Let $R$ be a ring with identity and $R$-mod the category of all unital left $R$ modules. A functor $\sigma: R$-mod $\rightarrow R$-mod is called a preradical if $\sigma(M)$ is a submodule of $M$ for each $M \in R$-mod and $\sigma(M) \alpha \subseteq \sigma(N)$ for each morphism $\alpha: M \rightarrow N$ in $R$-mod. A preradical $\sigma$ is called an idempotent preradical (resp. a radical) if $\sigma(\sigma(M))=\sigma(M)($ resp. $\sigma(M / \sigma(M))=0)$ for all $M \in R$-mod. A preradical is called left exact (resp. cohereditary) if it is kernel preserving (resp. epi-preserving). Every left exact (resp. cohereditary) preradical is idempotent (resp. a radical). A preradical is called a cotorsion radical (resp. an exact radical) if it is an idempotent cohereditary radical (resp. a left exact cohereditary radical). For preradicals $\sigma_{1}$ and $\sigma_{2}, \sigma_{1} \leq \sigma_{2}$ means that $\sigma_{1}(M) \subseteq \sigma_{2}(M)$ for all $M \in R$-mod.

To a preradical $\sigma$ for $R$-mod, we associate the pair $\left(\mathscr{I}_{\sigma}, \mathscr{F}_{\sigma}\right)$ of classes of Received September 8, 1980. 
modules in $R$-mod given by

$$
\mathscr{I}_{\sigma}=\left\{{ }_{R} X \mid \sigma(X)=X\right\} \text { and } \mathscr{F}_{\sigma}=\left\{{ }_{R} X \mid \sigma(X)=0\right\} .
$$

A class $\mathcal{C}$ of modules is called a pretorsion class if it is closed under quotients and direct sums, and is called a pretorsion-free class if it is closed under submodules and direct products. It is known that the assignment $\sigma \mapsto \mathscr{I}_{\sigma}$ is a bijection between idempotent preradicals for $R$-mod and pretorsion classes of modules, under which left exact preradicals correspond to pretorsion classes closed under submodules ([16, Chap. 6]). Dually, the assignment $\sigma \mapsto \mathscr{F}_{\sigma}$ is a bijection between radicals for $R$-mod and pretorsion-free classes of modules, under which cohereditary radicals correspond to pretorsion-free classes closed under quotients.

A class $\mathscr{I}$ of modules is called a torsion class if it is a pretorsion class closed under extentions. A class $\mathscr{I}$ of modules is called a torsion-free class if it is a pretorsion-free class closed under extensions. If $\sigma$ is an idempotent radical for $R$-mod, then $\mathscr{T}_{\sigma}$ is a torsion class and $\mathscr{F}_{\sigma}$ is a torsion-free class. Moreover the pair $\left(\mathscr{I}_{\sigma}, \mathscr{I}_{\sigma}\right)$ forms a torsion theory for $R$-mod in the sense of [3]. It is well known that, under the above assignments, we have bijective correspondences between: (1) idempotent radicals for $R$-mod, (2) torsion classes and (3) torsionfree classes. Finally, we remark that the assignment $\sigma \mapsto \mathscr{F}_{\sigma}$ is a bijection between cotorsion radicals for $R$-mod and torsion torsion-free (TTF-) classes, under which exact radicals correspond to $T T F$-classes closed under injective hulls.

For a class $\mathcal{C}$ of modules in $R$-mod, we define an idempotent preradical $t_{\mathcal{C}}$ for $R$-mod by

$$
t_{C}(M)=\Sigma\left\{\operatorname{Im}(\alpha) \mid \alpha \in \operatorname{Hom}_{R}(Q, M), Q \in \mathcal{C}\right\}
$$

for each $M \in R$-mod. In general $\mathcal{C}$ is not a set. An accurate treatment of $t_{C}(M)$ was given by K. Ohtake. Put $\mathcal{S}=\left\{\mathcal{C}^{\prime} \subseteq \mathcal{C} \mid \mathcal{C}^{\prime}\right.$ is a set $\}$. Let $\mathscr{I}=\left\{t_{C^{\prime}}(M) \mid \mathcal{C}^{\prime} \in \mathcal{S}\right\}$. Then $\mathscr{I}$ is a set and so $t_{C}(M)$ is defined via $\Sigma\left\{t_{C^{\prime}}(M) \mid t_{C^{\prime}}(M) \in \mathscr{I}\right\} . t_{C}$ is a unique minimal one of those preradicals $t$ for $R$-mod satisfying $t(Q)=Q$ for all $Q \in \mathcal{C}$. If $\mathcal{C}=\{Q\}$ is a singleton, we write $t_{Q}$ for $t_{C}$. Some basic properties of $t_{Q}$ are discussed in [8]. Dually, for a class $\mathscr{D}$ of modules in $R$-mod, we define a radical $k_{\mathscr{D}}$ for $R$-mod by

$$
k_{\mathscr{D}}(M)=\bigcap\left\{\operatorname{Ker}(\alpha) \mid \alpha \in \operatorname{Hom}_{R}(M, Q), Q \in \mathscr{D}\right\}
$$

for each $M \in R$-mod. $\quad k_{\mathscr{D}}$ is a unique maximal one of those preradicals $k$ for $R$ $\bmod$ satisfying $k(Q)=0$ for all $Q \in \mathscr{D}$. If $\mathscr{D}=\{Q\}$ is a singleton, we write $k_{Q}$ for $k_{\mathscr{D}}$. Some bacic properties of $k_{Q}$ are discussed in [9].

LEMMA 1.1. If $t$ is an idempotent preradical for $R$-mod, then there exists a 
class $\mathcal{C}$ of modules in $R$-mod such that $t=t_{C}$. Dually, if $k$ is a radical for $R$-mod, then there exists a class $\mathscr{D}$ of modules in $R$-mod such that $k=k_{\mathscr{D}}$.

Proof. Put $\mathcal{C}=\mathscr{I}_{t}$ and $\mathscr{D}=\mathscr{I}_{k}$.

Now we assume that ${ }_{R} P$ is a progenerator ( $=$ a finitely generated projective generator) in $R$-mod. We put $S=\operatorname{End}_{R}(P)$ and $P^{*}=\operatorname{Hom}_{R}(P, R)$. For a preradical $\sigma$ for $R$-mod, we associate the pair $(\mathscr{I}, \mathscr{F})$ of classes of modules in $S$-mod defined by

$$
\mathscr{I}=\left\{{ }_{S} Y \mid P \otimes_{S} Y \in \mathscr{I}_{\sigma}\right\} \quad \text { and } \mathscr{F}=\left\{{ }_{S} Y \mid P \otimes_{S} Y \in \mathscr{F}_{\sigma}\right\} .
$$

Since $P_{S}$ is also a progenerator in $\bmod -S, P \otimes_{S}(): S-\bmod \rightarrow R-\bmod$ is an exact functor that commutes with direct sums and direct products of modules. Thus, if $\sigma$ is an idempotent preradical for $R$-mod, then $\mathscr{I}=\mathscr{I}_{\tau}$ for some idempotent preradical $\tau$ for $S$-mod. Dually, if $\sigma$ is a radical for $R$-mod, then $\mathscr{F}=\mathscr{F}_{\tau}$ for some radical $\tau$ for $S$-mod. Using $P \otimes_{S} P^{*} \cong R$, we obtain the following propositions.

PROPOSITION 1.2. The asssgnment $\sigma \mapsto \tau$ where $\mathscr{I}_{\tau}=\left\{_{S} Y \mid P \otimes_{S} Y \in \mathscr{I}_{\sigma}\right\}$ is an order preserving bijection between idempotent preradicals for $R$-mod and those for $S$-mod, under which left exact preradicals for $R$-mod correspond to those for $S$-mod.

PROPOSITION 1.3. The assignment $\sigma \mapsto \tau$ where $\mathscr{F}_{\tau}=\left\{{ }_{S} Y \mid P \otimes_{S} Y \in \mathscr{F}_{\sigma}\right\}$ is an order preserving bijection between radicals for $R$-mod and those for $S$-mod, under which cohereditary radicals for $R$-mod correspond to those for $S$-mod.

It is easy to verify that, if $\sigma$ is an idempotent radical for $R$-mod, then the pair $\left(\mathscr{I}_{\tau}, \mathscr{I}_{\tau}\right)$ of classes of modules forms a torsion theory for $S$-mod, where $\mathscr{I}_{\tau}$ $=\left\{{ }_{S} Y \mid P \otimes_{S} Y \in \mathscr{I}_{\sigma}\right\}$ and $\mathscr{F}_{\tau}=\left\{{ }_{S} Y \mid P \otimes_{S} Y \in \mathscr{F}_{\sigma}\right\}$. Hence we have the coincidence of assignments $\sigma \mapsto \mathscr{I}_{\tau} \mapsto \tau$ and $\sigma \mapsto \mathscr{F}_{\tau} \mapsto \tau$.

Proposition 1.4. The assignment $\sigma \mapsto \tau$ is an order preserving bijection between idempotent radicals for $R$-mod and those for $S$-mod, under which left exact radicals for $R$-mod correspond to those for $S$-mod, cotorsion radicals for $R$-mod correspond to those for $S$-mod and exact radicals for $R$-mod correspond to those for $S$-mod.

Recall that the assignment $\sigma \mapsto \sigma(R)$ is a bijection between cohereditary radicals for $R$-mod and ideals of $R$, under which cotorsion radicals for $R$-mod correspond to idempotent ideals of $R$. In [7], it is proved that if $\sigma$ is a cotorsion radical for $R$-mod with associated idempotent ideal $L$ of $R$, and if $\tau$ is the 
corresponding cotorsion radical for $S$-mod with associated idempotent ideal $J$ of $S$, then $J=\{s \in S \mid P s \subseteq L P\}$.

We refer to [4, Chap. 2] some Morita invariant properties around left exact radicals. In particular it is shown in [4, Prop. 9.4] that, if $\sigma$ is a left exact radical for $R$-mod such that $\sigma(R)=0$, and if $\tau$ is the corresponding left exact radical for $S$-mod, then $\tau(S)=0$. The argument of the proof is valid for proving the first part of the next proposition.

PROPOSITION 1.5. If $\sigma$ is a radical for $R$-mod such that $\sigma(R)=0$, and if $\tau$ is the corresponding radical for $S$-mod, then $\tau(S)=0$. The same holds for an idempotent preradical.

PROOF. Let $\sigma$ be an idempotent preradical for $R$-mod such that $\sigma(R)=0$, and $\tau$ the corresponding idempotent preradical for $S$-mod. Assume $\tau(S) \neq 0$. Then we have a nonzero homomorphism $h: P \otimes_{S} \tau(S) \rightarrow P \otimes_{S} S \cong P$. Since ${ }_{R} P$ is torsionless, for any nonzero $u \in \operatorname{Im}(h)$, there exists a $g \in P^{*}$ satisfying $(u) g \neq 0$. Thus $0 \neq$ $(u) g \in \operatorname{Im}(h \circ g)$. Since $P \bigotimes_{S} \tau(S) \in I_{\sigma}$, we have $(u) g \in \sigma(R)$, which is impossible because $\sigma(R)=0$.

\section{A table of preradicals}

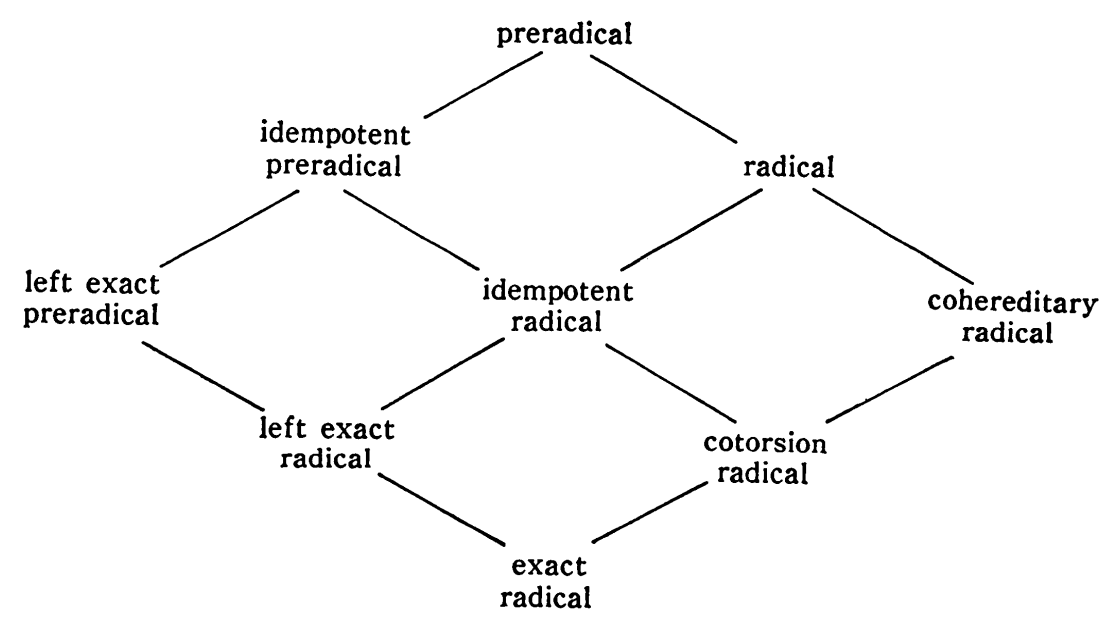

\section{Simple rings.}

We call an ideal $I$ of $R$ a preradical ideal if there exists a preradical $\sigma$ for $R$-mod such that $\sigma(R)=I$. A preradical ideal of a left exact preradical (resp. a left exact radical) is nothing but a pretorsion ideal (resp. a torsion ideal) in the sense of [6]. From now on, we shall study the rings which have no non-trivial preradical ideals $\sigma(R)$, where we take $\sigma$ as an idempotent preradical (or an exact 
radical, etc) for $R$-mod, and give several characterizations of those rings. Note that, for a preradical $\sigma$ for $R$-mod, $\sigma(R)=R$ if and only if $\sigma=1$, where 1 stands for the identity functor for $R$-mod. Hence we may rephrase our question as: When the preradical ideals $\sigma(R)$ vanish for various types of preradicals $\sigma \neq 1$ for $R$-mod? To begin with, we have

PROPOSITION 2.1. The following properties are equivalent for a ring $R$ :

(1) $\sigma(R)=0$ for every preradical $\sigma \neq 1$ for $R$-mod.

(2) $\sigma(R)=0$ for every radical $\sigma \neq 1$ for $R$-mod.

(3) $\sigma(R)=0$ for every cohereditary radical $\sigma \neq 1$ for $R$-mod.

(4) There exist only two cohereditary radicals for $R$-mod.

(5) $R$ is a simple ring (i.e. it has exactly two ideals).

(6) Every nonzero (cyclic) left R-module is faithful.

(7) $R K=R$ for every nonzero right ideal $K$ of $R$.

PROOF. Noting that each ideal of $R$ is a preradical ideal of a cohereditary radical for $R$-mod, we have the implications $(1) \Rightarrow(2) \Rightarrow(3) \Rightarrow(4) \Rightarrow(5)$.

$(5) \Rightarrow(6)$. Let $M(\neq 0)$ be a left $R$-module. Since $\operatorname{Ann}_{R}(M)$ is a proper ideal of $R$, we have $\operatorname{Ann}_{R}(M)=0$.

(6) $\Rightarrow(7)$. Assume $R \neq R K$ for some right ideal $K$ of $R$. For any $a \in K$, we have $a R \subseteq K \subseteq R K$. Therefore $a \in \mathrm{Ann}_{R}(R / R K)=0$ by the assumption. Hence we obtain $K=0$.

$(7) \Rightarrow(1)$. Assume $\sigma(R) \neq 0$ for some preradical $\sigma$ for $R$-mod. Then we have $R \sigma(R)=R$ by (7). Hence $\sigma(R)=R$, and so $\sigma=1$ as desired.

The vanishing of the preradical ideals $\sigma(R)$ of idempotent radicals (or left exact preradicals, left exact radicals) $\sigma \neq 1$ for $R$-mod has been characterized by several authors. We briefly summarize these results.

Definition AND TheOREM A ([2, Prop. 1.10]). The following properties are equivalent for a ring $R$ :

(1) $R$ is a left $R$-ring, i.e. $\sigma(R)=0$ for every idempotent radical $\sigma \neq 1$ for $R$-mod.

(2) $\operatorname{Hom}_{R}(I, R / I) \neq 0$ for every non-trivial left ideal $I$ of $R$.

(3) $\operatorname{Hom}_{R}(I, M) \neq 0$ for every nonzero left ideal $I$ of $R$ and nonzero $M \in$ $R$-mod.

Definition AND Theorem B ([6, p2], [14, Theorem 1.7], [17, Theorem 2.1] and [1, Prop. 3.2]). The following properties are equivalent for a ring $R$ : 
(1) $R$ is a left $S P$-ring, i.e. $\sigma(R)=0$ for every left exact preradical $\sigma \neq 1$ for $R$-mod.

(2) Every nonzero left ideal of $R$ is cofaithful.

(3) Every nonzero left ideal of $R$ generates $E\left({ }_{R} R\right)$.

(4) $R$ is a left non-singular prime ring, and every non-singular quasi-injective left $R$-module is injective.

Definition AND TheOREM C ([2, Theorem 2.4] and [6, p91]). The following properties are equivalent for a ring $R$ :

(1) $R$ is a left CTF-ring, i.e. $\sigma(R)=0$ for every left exact radical $\sigma \neq 1$ for $R$-mod.

(2) For every non-trivial left ideal $I$ of $R$, there exist $x \in I, y \in R \backslash I$ such that $(0: x) \subseteq(I: y)$.

(3) Every nonzero injective left $R$-module is faithful.

\section{Left $G$-rings.}

Remark that if a module ${ }_{R} M$ is a generator for $R$-mod, then the dual $\operatorname{Hom}_{R}(M, R) \neq 0$. The next theorem deals with a ring $R$ satisfying the converse statement.

THEOREM 3.1. The following properties are equivalent for a ring $R$ :

(1) $\sigma(R)=0$ for every idempotent preradical $\sigma \neq 1$ for $R$-mod.

(2) Every left $R$-module with nonzero dual is a generator for $R$-mod.

(3) Every nonzero torsionless left $R$-module is a generator for $R$-mod.

(4) Every nonzero submodule of a projective left $R$-module is a generator for $R$-mod.

(5) Every nonzero left ideal of $R$ is a generator for $R$-mod.

(6) Every nonzero ideal of $R$ is a generator for $R$-mod.

PROOF. For a module ${ }_{R} Q$, one can verify that $t_{Q}=1$ if and only if ${ }_{R} Q$ is a generator for $R$-mod.

$(1) \Rightarrow(2)$. If a module ${ }_{R} Q$ is not a generator for $R$-mod, then the idempotent preradical $t_{Q} \neq 1$. Therefore $t_{Q}(R)=0$ and so $\operatorname{Hom}_{R}(Q, R)=0$.

(2) $\Rightarrow(3)$. If ${ }_{R} Q$ is a nonzero torsionless module, then $\operatorname{Hom}_{R}(Q, R) \neq 0$ and so ${ }_{R} Q$ is a generator for $R$-mod.

$(3) \Rightarrow(4)$. This is clear from the facts that every projective module is torsionless and every submodule of a torsionless module is torsionless.

$(4) \Rightarrow(5) \Rightarrow(6)$. Clear. 
(6) $\Rightarrow(1)$. Let $\sigma$ be an idempotent preradical for $R$-mod. Assume $\sigma(R) \neq 0$. Since $\sigma(R)$ is a generator for $R$-mod, we have $t_{\sigma(R)}=1$. Recall that $t_{\sigma(R)}$ is a unique minimal one of those preradicals $t$ such that $t(\sigma(R))=\sigma(R)$. Hence we obtain $t_{\sigma(R)} \leq \sigma$, and so $\sigma=1$.

Definition 3.2. A ring which satisfies one of the equivalent conditions of Theorem 3.1 is called a left G-ring.

COROLlary 3.3. A property that a ring is a left G-ring is Morita invariant.

Proof. This is clear by (4) of Theorem 3.1 or (1) of Theorem 3.1 combined with Proposition 1.5.

In [11, Theorem 4.15], it is proved that, for a ring $R$, every nonzero (simple) left $R$-module is a generator for $R$-mod if and only if $R$ is simple artinian.

COROLlary 3.4. $R$ is a left $G$-ring with nonzero (left) socle if and only if $R$ is simple artinian.

Proof. Let $R$ be a left $G$-ring with socle $S \neq 0$. Then $S$ generates ${ }_{R} R$, and so $R$ is completely reducible. But since $R$ is prime by Theorem $\mathrm{B}, R$ is simple artinian.

In [13, Theorem 1.2], it is proved that, every nonzero left ideal of $R$ is a progenerator for $R$-mod if and only if $R$ is left hereditary left noetherian prime ring without non-trivial idempotent ideals.

COROLLARY 3.5. If $R$ is left hereditary, $R$ is a left $G$-ring if and only if every nonzero projective left $R$-module is a generator for $R$-mod.

PRoof. This is clear by (4) of Theorem 3.1.

COROLLARY 3.6. Every left G-ring is a left SP-ring. The converse holds if $R$ is left self-injective. Also every left $G$-ring is a left $R$-ring.

Proof. This is clear by Theorem 3.1 and Theorems A and B.

Proposition 3.7. If $R$ is a left $G$-ring, then the maximal left ring of quotients $Q_{\max }$ of $R$ is simple and left self-injective. In particular $Q_{\max }$ is also a left $G$-ring. If $R$ is a left $G$-ring and the classical left ring of quotients $Q_{\mathrm{cl}}$ of $R$ exists, then $Q_{\mathrm{cl}}$ is also a left $G$-ring.

ProOF. The first part follows from the fact that $Q_{\max }$ is simple and left 
self-injective if $R$ is a left $S P$-ring [6, Prop. 6.2].

Now let $A$ be a nonzero left ideal of $Q_{\mathrm{cl}}$. Since $A \cap R$ is a nonzero left ideal of $R, A \cap R$ generates ${ }_{R} R$. Thus there exist $R$-homomorphisms $f_{i}: A \cap R \rightarrow R$ $(i=1, \cdots, m)$ such that $\sum_{i=1}^{m} f_{i}: \bigoplus_{i=1}^{m}(A \cap R) \rightarrow R$ is an $R$-epimorphism. For each $i, f_{i}$ induces a $Q_{\mathrm{cl}}$-homomorphism $g_{i}: Q_{\mathrm{cl}}(A \cap R) \rightarrow Q_{\mathrm{cl}}$ defined by $\left(\sum_{j=1}^{n} q_{j} x_{j}\right) g_{i}=\sum_{j=1}^{n} q_{j}\left(x_{j}\right) f_{i}$ where $q_{j} \in Q_{\mathrm{cl}}$ and $x_{j} \in A \cap R$. Since $Q_{\mathrm{cl}}(A \cap R)=A$, we have a $Q_{\mathrm{cl}}$-epimorphism $\sum_{i=1}^{m} g_{i}: \bigoplus_{i=1}^{m} A \rightarrow \sum_{i=1}^{m} Q_{\mathrm{cl}} \operatorname{Im}\left(f_{i}\right)=Q_{\mathrm{cl}}\left(\sum_{i=1}^{m} \operatorname{Im}\left(f_{i}\right)\right)=Q_{\mathrm{cl}} R=Q_{\mathrm{cl}}$. Hence $A$ is a generator for $Q_{\mathrm{cl}-\text { mod. }}$

EXAMPLE 3.8. Every simple ring is a left $G$-ring, but the converse is not true. For a counter example, we may take the ring $Z$ of integers. The ring $Z_{n}$ of $n \times n$ matrices over $Z$ is a left and right $G$-ring by using Corollary 3.3, which is not simple.

EXAmple 3.9. Every left $G$-ring is a left $R$-ring, but the converse is not true. For a counter example, we may take the ring $R=Z /\left(p^{n}\right)$, where $p$ is a prime and $n$ is an integer greater than 1 . To prove $R$ is a (left) $R$-ring, we verify that $R$ satisfies (2) of Theorem A. For any non-trivial ideal $I=\left(p^{i}\right) /\left(p^{n}\right)$ where $i=1, \cdots, n-1$, we define $j=0$ if $2 i-n \leq 0$ and $j=2 i-n$ if $2 i-n>0$. Then the correspondence $p^{i}+\left(p^{n}\right) \mapsto\left(p^{j}+\left(p^{n}\right)\right)+I$ is a nonzero $R$-homomorphism from $I$ to $R / I$. Now remark that $R$ has the nonzero socle $\left(p^{n-1}\right) /\left(p^{n}\right)$ but $R$ is not simple artinian. Hence $R$ is not a (left) $G$-ring by Corollary 3.4. We remark, by this example, a factor ring of a left $G$-ring need not be a left $G$-ring.

\section{Some generalizations of left $G$-rings.}

LEMmA 4.1. Let $I=\sum_{i=1}^{m} R a_{i}$ be a finitely generated left ideal of $R$. Then $I$ is a generator for $R$-mod if and only if there exist subsets $\left\{b_{i j} \mid i=1, \cdots, m\right.$; $j=1, \cdots, n\}$ and $\left\{s_{i j} \mid i=1, \cdots, m ; j=1, \cdots, n\right\}$ of $R$ such that (1) $\sum_{i=1}^{m} r_{i} a_{i}=0$ implies $\sum_{i=1}^{m} r_{i} b_{i j}=0$ for $\left\{r_{i} \mid i=1, \cdots, m\right\} \subseteq R$ and $j=1, \cdots, n$ and (2) $\sum_{j=1}^{n} \sum_{i=1}^{m} s_{i j} b_{i j}$ $=1_{R}$.

Proof. Put $K=\left\{\left(b_{1}, \cdots, b_{m}\right) \in R^{m} \mid \sum_{i=1}^{m} r_{i} a_{i}=0\right.$ implies $\sum_{i=1}^{m} r_{i} b_{i}=0$ for $r_{1}, \cdots$, $\left.r_{m} \in R\right\}$. Then the correspondence $f \mapsto\left(\left(a_{1}\right) f, \cdots,\left(a_{m}\right) f\right)$ is a bijection between $\operatorname{Hom}_{R}(I, R)$ and $K$. Now ${ }_{R} I$ is a generator for $R$-mod if and only if there exists 
a subset $\left\{f_{j} \mid j=1, \cdots, n\right\}$ of $\operatorname{Hom}_{R}(I, R)$ such that $\sum_{j=1}^{n}(I) f_{j}=\sum_{j=1}^{n} \sum_{i=1}^{m} R\left(a_{i}\right) f_{j}=R$. This is equivalent to the existence of a subset $\left\{\left(b_{1 j}, \cdots, b_{m j}\right) \mid j=1, \cdots, n\right\}$ of $K$ such that $\sum_{j=1}^{n} \sum_{i=1}^{m} R b_{i j}=R$.

PROPOSITION 4.2. The following properties are equivalent for a ring $R$ :

(1) Every nonzero finitely generated left ideal of $R$ is a generator for $R$-mod.

(2) Every finitely generated left $R$-module with nonzero dual is a generator for $R$-mod.

(3) Every nonzero finitely generated torsionless left $R$-module is a generator for $R$-mod.

(4) Every nonzero finitely generated submodule of a projective left $R$-module is a generator for $R$-mod.

Proof. $(1) \Rightarrow(2)$. Let $M$ be a finitely generated left $R$-module with an $f(\neq 0) \in \operatorname{Hom}_{R}(M, R)$. Then the finitely generated left ideal $\operatorname{Im}(f)$ generates ${ }_{R} R$, and so $M$ generates ${ }_{R} R$.

(2) $\Rightarrow(3) \Rightarrow(4) \Rightarrow(1)$. Clear.

DEFINITION 4.3. A ring which satisfies one of the equivalent conditions of Proposition 4.2 is called a left FGG-ring.

COROLLARY 4.4. If $R$ is left semihereditary, $R$ is a left FGG-ring if and only if every nonzero finitely generated projective left $R$-module is a generator for $R$-mod.

PROPOSITION 4.5. The following properties are equivalent for a ring $R$ :

(1) Every nonzero cyclic left ideal of $R$ is a generator for $R$-mod.

(2) $R a^{l r}=R$ for every nonzero $a \in R$, where $a^{l r}=\operatorname{Ann}_{R}^{r}\left(\operatorname{Ann}_{R}^{l}(a)\right)$.

(3) $R K=R$ for every nonzero annihilator right ideal $K$ (i. e. $K=\operatorname{Ann}_{R}^{r}(X)$ for some subset $X$ of $R$ ) of $R$.

(4) Every cyclic left $R$-module with nonzero dual is a generator for $R$-mod.

(5) Every nonzero cyclic torsionless left $R$-module is a generator for $R$-mod.

(6) Every nonzero cyclic submodule of a projective left $R$-module is a generator for $R$-mod.

Proof. (1) $\Rightarrow(2)$. By using Lemma 4.1, for a nonzero cyclic left ideal $I=R a$ of $R, I$ is a generator for $R$-mod if and only if there exist subsets $\left\{b_{1}, \cdots, b_{n}\right\}$ of $a^{l r}$ and $\left\{s_{1}, \cdots, s_{n}\right\}$ of $R$ such that $\sum_{j=1}^{n} s_{j} b_{j}=1_{R}$, or equivalently, $R a^{l r}=R$. 
(2) $\Rightarrow(3)$. Let $K$ be a nonzero annihilator right ideal of $R$. For a nonzero $a \in K, a^{l r} \subseteq K$ implies $R K=K$ by the assumption (2).

(3) $\Rightarrow(4)$. Let $L$ be a left ideal of $R$ such that $\operatorname{Hom}_{R}(R / L, R) \neq 0$. Then we have $L^{r} \neq 0$. For every $c \in L^{r}$, we define $\xi_{c} \in \operatorname{Hom}_{R}(R / L, R)$ as $\xi_{c}(x+L)=x c$. Now consider an $R$-homomorphism $\xi=\Sigma \xi_{c}: \bigoplus_{c \in L^{r}}(R / L) \rightarrow R$. Then $\operatorname{Im}(\xi)=R L^{r}=R$ by (3). Thus ${ }_{R}(R / L)$ is a generator for $R$-mod.

$(4) \Rightarrow(5) \Rightarrow(6) \Rightarrow(1)$. Clear.

DEFINITION 4.6. A ring which satisfies one of the equivalent conditions of Proposition 4.5 is called a left $C G$-ring.

Proposition 4.7. The following properties are equivalent for a ring $R$ :

(1) $R$ is a left FGG-ring.

(2) For each positive integer $n$, the ring $R_{n}$ of $n \times n$ matrices over $R$ is a left $C G$-ring.

Proof. (1) $\Rightarrow(2)$. By Proposition 4.2, we see that a property "left FGG" is Morita invariant.

$(2) \Rightarrow(1)$. Let $I$ be any nonzero finitely generated left ideal of $R$, say $I=$ $R a_{1}+\cdots+R a_{n}$. In $R_{n}$ we put $\omega=\left(a_{i j}\right)$ where $a_{i 1}=a_{i}$ and all other entries are zero. By the assumption, for some $k$, we have an $R_{n}$-epimorphism $\bigoplus_{i=1}^{k} R_{n} \omega \rightarrow R_{n}$, which is in fact an $R$-epimorphism under the change of rings $R \rightarrow R_{n}$ (canonical map). Since $R_{n} \omega \cong \bigoplus_{j=1}^{n} I$, there exists an $R$-epimorphism $\bigoplus_{i=1}^{k} \bigoplus_{j=1}^{n} I \rightarrow R_{n}$. Combining this with an $R$-epimorphism ${ }_{R}\left(R_{n}\right) \rightarrow_{R} R\left(\left(c_{i j}\right) \mapsto c_{11}\right)$, we obtain a desired $R$-epimorphism $\bigoplus_{i=1}^{k} \bigoplus_{j=1}^{n} I \rightarrow R$.

Now we consider another generalization of left $G$-rings. Recall that a semiprime ring is characterized as a ring whose essential left ideals are faithful. In [5], D. Handelman studied the structure of left strongly semiprime (SSP-)rings (i. e. rings whose essential left ideals are cofaithful). Among others, a ring $R$ is left $S S P$ if and only if $R$ is a finite subdirect product of left $S P$-rings. So we shall consider a ring $R$ whose essential left ideals are generators for $R$-mod.

PROPOSITION 4.8. The following properties are equivalent for a ring $R$ :

(1) Every essential left ideal of $R$ is a generator for $R$-mod.

(2) Every ideal which is essential in $R$ as a left ideal is a generator for $R$-mod. 
(3) Every module ${ }_{R} Q$ satisfying that $t_{Q}(R)$ is an essential left ideal is a generator for $R$-mod.

Proof. $\quad(1) \Rightarrow(2)$. Clear.

$(2) \Rightarrow(3)$. Assume, for a module ${ }_{R} Q, t_{Q}(R)$ is essential in $R$ as a left ideal. Since $Q$ generates $t_{Q}(R)$ and $t_{Q}(R)$ generates ${ }_{R} R, Q$ is a generator for $R$-mod.

(3) $\Rightarrow(1)$. Let $K$ be an essential left ideal of $R$. Clearly we have $K \subseteq t_{K}(R)$ $\subseteq R$, and so $t_{K}(R)$ is essential in $R$ as a left ideal. By (3), $K$ is a generator for $R$-mod.

DEFINITION 4.9. A ring which satisfies one of the equivalent conditions of Proposition 4.8 is called a left EG-ring.

Proposition 4.10. (1) Every ring direct summand of a left EG-ring is a left EG-ring.

(2) Every finite direct sum of left EG-rings is a left EG-ring.

Proof. (1). Let $T=R \oplus S$ be a ring decomposition of a left $E G$-ring $T$. To prove $R$ is a left $E G$-ring, let $A$ be any essential left ideal of $R$. It is easy to verify that ${ }_{T}(A \oplus S)$ is essential in $T$. Hence for some $n$, there exist a $T$ epimorphism $\bigoplus_{i=1}^{n}(A \oplus S) \rightarrow T$, which is also an $R$-epimorphism. The projection map $T \rightarrow R$ is also an $R$-epimorphism. Composing these epimorphisms, we have an $R$ epimorphism $\bigoplus_{i=1}^{n}(A \oplus S) \rightarrow T \rightarrow R$. Now remark that every $R$-homomorphism $f$ : $A \oplus S \rightarrow R$ vanishes $S$, becanse $R((0, S) f)=(R(0, S)) f=(0,0) f=0$. Hence we have an $R$-epimorphism $\bigoplus_{i=1}^{n} A \rightarrow R$.

(2). Let $R=\bigoplus_{i=1}^{n} R_{i}$ be a direct sum of left $E G$-rings $R_{i}(i=1, \cdots, n)$. For each $i$, we regard the projection map $\pi_{i}: R \rightarrow R_{i}$ as $R$-homomorphism. Then for any essential left ideal $I$ of $R,(I) \pi_{i}$ is an essential left ideal and also is an $R$ submodule of $R_{i}$. By the assumption, for some $k_{i}$. there exists an $R_{i}$-epimorphism $\bigoplus_{j=1}^{k_{i}}(I) \pi_{i} \rightarrow R_{i}$ which is also an $R$-epimorphism. Combining this with an $R$-epimorphism $I \rightarrow(I) \pi_{i}$, we have an $R$-epimorphism $\bigoplus_{j=1}^{k_{i}} I \rightarrow R_{i}$. Hence we have a desired $R$-epimorphism $\bigoplus_{i=1}^{n} \bigoplus_{j=1}^{k_{i}} I \rightarrow \bigoplus_{i=1}^{n} R_{i}=R$.

Note that an infinite direct product of left $E G$-rings need not be a left $E G$ ring. For example, let $K$ be a field and $R=\prod_{i=1}^{\infty} K$. Then $I=\bigoplus_{i=1}^{\infty} K$ is an essential ideal of $R$, but is not cofaithful, and so $I$ is not a generator for $R$-mod. 
Proposition 4.11. If $R$ is a left EG-ring and the classical left ring of quotients $Q_{\mathrm{cl}}$ of $R$ exists, then $Q_{\mathrm{cl}}$ is also a left EG-ring.

Proof. Let $A$ be an essential left ideal of $Q_{\mathrm{cl}}$. It is easy to verify that $A \cap R$ is an essential left ideal of $R$. Hence $A \cap R$ is a generator for $R$-mod, and so $A$ is a generator for $Q_{\mathrm{cl}}$-mod by the same argument of the proof of Proposition 3.7 .

EXAMPLE 4.12. Every left $G$-ring is a left $F G G$-ring. Every left $F G G$-ring is a left $C G$-ring, but the converse is not true. In fact, we shall give an example of a left $C G$-ring $R$ having a finitely generated essential left ideal which is not a generator for $R$-mod. Let $R=K[x, y]$ be a polynomial ring over a field $K$. Since $R$ is a domain, every nonzero cyclic (left) ideal of $R$ is isomorphic to $R$. Thus $R$ is a (left) $C G$-ring. Now let $I=(x, y)$ be an ideal generated by $x$ and $y$. Remark that $I$ is essential in $R$. We claim, for every $R$-homomorphism $f: I \rightarrow R$, there exists an $r^{*} \in R$ such that $(z) f=z r^{*}$ for all $z \in I$. Put $(x) f=r$ and $(y) f=s$. Then $(x y) f=x s=y r$, and so there exists an $r^{*} \in R$ such that $r=x r^{*}$ and $s=y r^{*}$. Thus for every $z=u x+v y \in I$ where $u$ and $v$ are elements of $R,(z) f=u r+v s=$ $(u x+v y) r^{*}=z r^{*}$ as desired. Hence for every $f \in \operatorname{Hom}_{R}(I, R)$, we have $\operatorname{Im}(f) \subseteq I$, proving that $I$ is not a generator for $R$-mod.

EXAMPlE 4.13. Every left $C G$-ring is a left $S P$-ring, but the converse is not true. Let $D=Z_{2}\left[x_{1}, x_{2}, x_{3}, \cdots\right]$ be the free non-commuting $Z_{2}$-algebra on $x_{i}$ $(i=1,2,3, \cdots)$. Let $I$ be the two-sided ideal in $D$ generated by monomials of the form $x_{i} x_{j} x_{k}$ with $i<j<k$. As is shown in [6, p9], $R=D / I$ is a left $S P$-ring. Now we show that a cyclic left ideal $A=\left(D x_{3}+I\right) / I$ of $R$ is not a generator for $R$-mod. For every $R$-homomorphism $f:{ }_{R} A \rightarrow{ }_{R} R$, we put $\left(x_{3}+I\right) f=m+I$, where $m \in D$. Let $m=m_{1}+\cdots+m_{p}$ be a sum of (distint) monomials in $D$. Since $x_{1} x_{2} x_{3}$ $\in I$, we have $x_{1} x_{2} m=x_{1} x_{2}\left(m_{1}+\cdots+m_{p}\right) \in I$, and so $x_{1} x_{2} m_{i} \in I$ for $i=1, \cdots, p$. We may assume that each monomial $m_{i}=x_{j_{1}} x_{j_{2}} \cdots x_{j_{k}} \notin I$. Hence $j_{1}$ must be greater than 2 , and so $m \in \sum_{n=3}^{\infty} x_{n} D$. Therefore $\operatorname{Im}(f) \subseteq\left(\sum_{n=3}^{\infty} D x_{n} D+I\right) / I \neq R$, proving that $A$ is not a generator for $R$-mod.

EXAMPLE 4.14. Every left $G$-ring is a left $E G$-ring, but the converse is not true. In fact, $R=Z \oplus Z$ is a (left) $E G$-ring by Proposition 4.10 , but $R$ is not prime. One may expect that, if $R$ is a left $E G$-ring, then every (essential submodule of a) projective left $R$-module is a generator for $R$-mod. But this is not true. Once again let $R=Z \oplus Z$, and put $I=(Z, 0)$ be an ideal of $R$. Clearly ${ }_{R} I$ is 
projective, but an easy verification shows that $t_{I}(R)=I$, which means ${ }_{R} I$ is not a generator for $R$-mod.

Proposition 4.15. A ring $R$ is left $G$ if and only if $R$ is both left $E G$ and left $R$.

Proof. $\quad(\Rightarrow)$ : This was done in Example 4.14 and Corollary 3.6. $(\Leftarrow)$ : For every idempotent preradical $\sigma$ for $R$-mod satisfying $\sigma(R) \neq 0$, we shall show that $\sigma=1$. Put $\bar{\sigma}$ be the smallest radical larger than $\sigma$ ([16, p137]). Since $R$ is a left $R$-ring, we have $\bar{\sigma}=1$. We claim that $\sigma(R)$ is essential in ${ }_{R} R$. Let $A$ be a left ideal such that $A \cap \sigma(R)=0$. Then $\sigma(A) \subseteq A \cap \sigma(R)=0$, and so $A \in \mathscr{F}_{\sigma}=\mathscr{F}_{\bar{\sigma}}=\{0\}$ because $\bar{\sigma}=1$. Now since $R$ is a left $E G$-ring, $\sigma(R)$ generates ${ }_{R} R$. Thus for some $n$ and a module ${ }_{R} N, \bigoplus_{i=1}^{n} \sigma(R)=R \oplus N$. Since $\sigma$ is idempotent, we also have $\bigoplus_{i=1}^{n} \sigma(R)=\sigma(R) \oplus \sigma(N)$. Therefore $\sigma(R)=R$ which means $\sigma=1$ as desired.

\section{Rings without non-trivial left strongly idempotent ideals (left E2-rings).}

PROPOSITION 5.1. The following properties are equivalent for a ring $R$ :

(1) $\sigma(R)=0$ for every cotorsion radical $\sigma \neq 1$ for $R$-mod.

(2) There exist only two cotorsion radicals for $R$-mod.

(3) $R$ has no non-trivial idempotent ideals.

Proof. Clear.

By using Proposition 1.4, we observe that the above property is Morita invariant.

EXAMPLE 5.2. Every left $R$-ring has no non-trivial idempotent ideals, but the converse is not true. For a counter example, consider $S=Z \times Q$, where $Z$ is the ring of integers and $Q$ the field of rational numbers. Define the addition on $S$ by component wise and the multiplication on $S$ by

$$
\left(z_{1}, q_{1}\right) *\left(z_{2}, q_{2}\right)=\left(z_{1} z_{2}, z_{1} q_{2}+z_{2} q_{1}\right) .
$$

Then $S$ becomes a commutative ring without non-trivial idempotent ideals, but as is shown in [2, Example 1.16] $S$ is not an $R$-ring.

Definition 5.3. We shall call that an ideal $I$ of a ring $R$ is left strongly idempotent, if $J=I J$ holds for every left ideal $J \subseteq I$.

Clearly every left strongly idempotent ideal is idempotent, but the converse 
is not true. For a counter example, let $R$ be the ring of $2 \times 2$ upper triangular matrices over a field $K$. One can check that $\left(\begin{array}{ll}0 & K \\ 0 & K\end{array}\right)$ is idempotent but not left strongly idempotent. On the other hand, $\left(\begin{array}{cc}K & K \\ 0 & 0\end{array}\right)$ is left strongly idempotent.

THEOREM 5.4. The following properties are equivalent for a ring $R$ :

(1) $\sigma(R)=0$ for every exact radical $\sigma \neq 1$ for $R$-mod.

(2) There exist only two exact radicals for $R$-mod.

(3) If a nonzero injective module ${ }_{R} E$ satisfies the condition that, for a left ideal $K, \operatorname{Hom}_{R}(R / K, E)=0$ implies $K+\operatorname{Ann}_{R}(E)=R$, then $E$ is faithful.

(4) There are no non-trivial ideals $I$ such that $I N=N \cap I M$ for each ${ }_{R} N \subseteq_{R} M$.

(5) There are no non-trivial (idempotent) ideals $I$ such that $(R / I)_{R}$ are flat.

(6) $R$ has no non-trivial left strongly idempotent ideals.

Proof. $\quad(1) \Leftrightarrow(2) . \quad$ Clear.

$(1) \Leftrightarrow(3)$. For a left exact radical $\sigma$ for $R$-mod, there exists an injective module ${ }_{R} E$ such that $\mathscr{I}_{\sigma}=\left\{R_{R} M \mid \operatorname{Hom}_{R}(M, E)=0\right\}$. In this case, $\sigma$ is an exact radical if and only if, for a left ideal $K, \operatorname{Hom}_{R}(R / K, E)=0$ implies $K+\sigma(R)=R$ ([15, Prop. 2.1]). Thus the equivalence of (1) and (3) is clear by noticing that $\sigma(R)=\mathrm{Ann}_{R}(E)$.

$(2) \Leftrightarrow(4)$. Clear.

(2) $\Leftrightarrow(5)$. Let $\sigma$ be a cotorsion radical for $R$-mod. It is well known (for example [12]) that $\sigma$ is left exact if and only if $(R / \sigma(R))_{R}$ is flat. Thus we have an equivalence of (1) and (5).

(5) $\Leftrightarrow(6)$. For an ideal $I,(R / I)_{R}$ is flat if and only if $I$ is a left strongly idempotent ideal ([10, Theorem 2]).

DEFINITION 5.5. A ring which satisfies one of the equivalent conditions of Theorem 5.4 is called a left E2-ring.

COROLlary 5.6. The property that a ring is a left E2-ring is Morita invariant.

PRoof. This is clear by (2) of Theorem 5.4 combined with Proposition 1.4.

COROLLARY 5.7. If $R$ is a left weakly regular ring (i.e. a ring whose left ideals are idempotent), then the following conditions are equivalent:

(1) $R$ is a simple ring.

(2) $R$ is a left E2-ring. 
PROOF. $\quad(1) \Rightarrow(2)$. Clear.

$(2) \Rightarrow(1)$. Let $I$ be a proper ideal of $R$. Since every left ideal is idempotent, $I$ is a left strongly idempotent ideal. Hence $I=0$ and so $R$ is simple.

EXAMPLE 5.8. There is a right E2-ring which is not a left E2-ring. Let $D=F[x, y]$ be the free non-commuting algebra on $\{x, y\}$ over a field $F$. Then $D x D=\bigoplus_{i=0}^{\infty} y^{i} x D \cong \bigoplus_{i=0}^{\infty} D_{D}$. The ring $R=$ End $\left(D x D_{D}\right)$ is right $S P$ (cf. [6, Example 13.2]) and so is right $E 2$. But $R$ contains a non-trivial left strongly idempotent ideal $K=\bigoplus_{i=0}^{\infty} e_{i} R$, where $e_{i}$ denotes the matrix with 1 in the $(i, i)$ position, 0 elsewhere.

EXAMPLE 5.9. If $R$ is a left $C T F$-ring, then every nonzero flat right $R$ module is faithful ([6, Prop. 13.9]). If $R$ has this property, then $R$ is left $E 2$ by (5) of Theorem 5.4. But the converse is not true. Let

$$
R=\left\{\left(\begin{array}{lll}
a & 0 & 0 \\
b & c & 0 \\
d & e & a
\end{array}\right) \mid a, b, c, d, e \in K\right\},
$$

where $K$ is a field. One can check that there are only two non-trivial idempotent ideals

$$
I_{1}=\left\{\left(\begin{array}{ccc}
a & 0 & 0 \\
b & 0 & 0 \\
d & e & a
\end{array}\right) \mid a, b, d, e \in K\right\} \quad \text { and } I_{2}=\left(\begin{array}{ccc}
0 & 0 & 0 \\
K & K & 0 \\
K & K & 0
\end{array}\right)
$$

Put $J_{1}=\left(\begin{array}{ccc}0 & 0 & 0 \\ K & 0 & 0 \\ K & K & 0\end{array}\right) \subseteq I_{1}$ and $J_{2}=\left(\begin{array}{ccc}0 & 0 & 0 \\ 0 & 0 & 0 \\ K & 0 & 0\end{array}\right) \subseteq I_{2}$. Then $J_{i} \neq I_{i} J_{i}(i=1,2)$. Thus $I_{i}$ $(i=1,2)$ are not left strongly idempotent ideals. This gives an example of left $E 2$-ring having non-trivial idempotent ideals. The same argument shows $R$ is also a right E2-ring. Now put

$$
A=\left(\begin{array}{ccc}
0 & 0 & 0 \\
K & K & 0 \\
0 & 0 & 0
\end{array}\right) \text { and } B=\left\{\left(\begin{array}{lll}
a & 0 & 0 \\
0 & 0 & 0 \\
d & e & a
\end{array}\right) \mid a, d, e \in K\right\}
$$

Then $R=A \oplus B$, and so $A_{R}$ is flat. But since $\operatorname{Ann}_{R}^{r}(A)=\left(\begin{array}{lll}0 & 0 & 0 \\ 0 & 0 & 0 \\ K & K & 0\end{array}\right) \neq 0, A_{R}$ is not faithful. Finally, we remark that $R$ is not semiprime.

EXAMPLE 5.10. We give an example of a prime ring which is not left $E 2$. Let $V_{D}$ be an infinite dimensional vector space over a division ring $D$. Put $R=$ 
End $\left(V_{D}\right)$. Then $R$ is a regular and prime ring. Put $I=\operatorname{soc}(R)$, then $I$ consists of $f \in R$ such that $\operatorname{Im}(f)_{D}$ is finite dimensional. Thus $I$ is a non-trivial (left) strongly idempotent ideal. One may remark that ${ }_{R} I$ is not cofaithful, and so $R$ is not a left $S S P$-ring.

PROPOSITION 5.11. If $R$ is a left E2-ring, then no non-trivial ideals of $R$ are direct summand as a right ideal.

ProOF. Let $I$ be a proper ideal and $K$ a right ideal such that $R=I \oplus K$. For every left ideal $J \subseteq I$, we have $K J \subseteq K I \subseteq I \cap K=0$, and so $I J=(I \oplus K) J=R J=J$. By the assumption, $I=0$ as desired.

EXAMPLE 5.12. By Proposition 5.11, every left E2-ring is indecomposable as a ring, but the converse is not true. For a counter example, we may take the ring of $2 \times 2$ upper triangular matrices over a field.

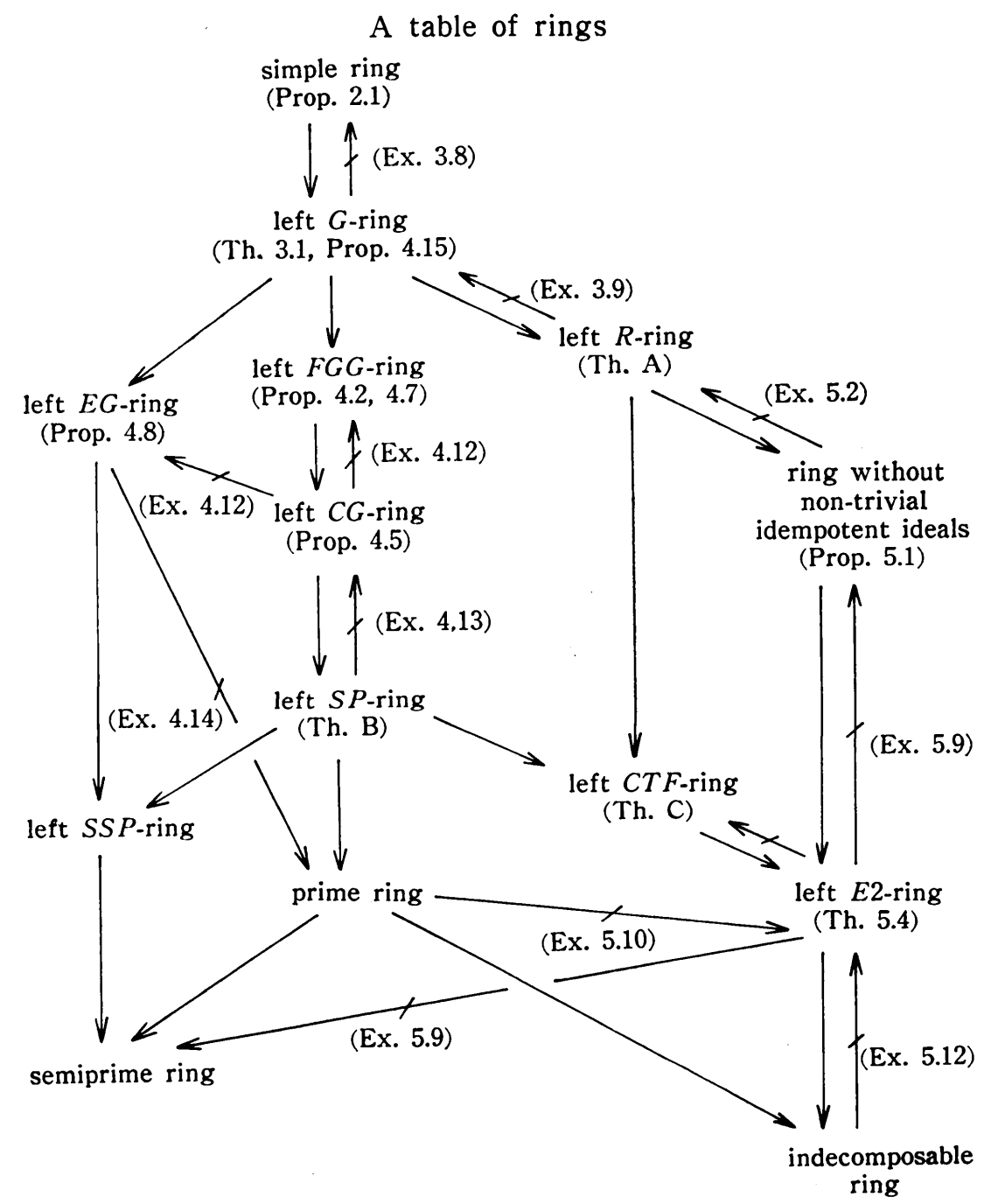




\section{Acknowledgement}

The author expresses his heartly thanks to Professor Y. Kurata for his various suggestions.

\section{References}

[1] Beachy, J.A. and Blair, W.D., Rings whose faithful left ideals are cofaithful. Pacific J. Math. 58 (1975), 1-13.

[2] Bican, L., Jambor, P., Kepka, T. and Nĕmec, P., On rings with trivial torsion parts. Bull. Austral. Math. Soc. 9 (1973), 275-290.

[3]. : Dickson, S.E., A torsion theory for abelian categories. Trans. Amer. Math. Soc. 121 (1966), 223-235.

[4] Golan, J.S., Localization of noncommutative rings. Marcel Dekker, Inc., New York (1975).

[5] Handelman, D., Strongly semiprime rings. Pacific J. Math. 60 (1975), 115-122.

[6] Handelman, D., Goodearl, K. R. and Lawrence, J., Strongly prime and completely torsionfree rings. Carleton Mathematical Series 109, Carleton University (1974).

[.7] Izawa, T., A remark on idempotent ideals of Morita equivalent rings. Rep. Fac. Sci., Shizuoka Univ. 13 (1979), 15-16.

[8] Katayama, H., On the cotorsion radicals. J. Fac. Liberal Arts, Yamaguchi Univ. 8 (1974), 239-243.

[9] Kurata, Y. and Katayama, H., On a generalization of $Q F-3^{\prime}$ rings. Osaka J. Math. 13 (1976), 407-418.

[10] Kiełpiński, R., Direct sums of torsions. Bull. Acad. Polon. Sci. 19 (1971), 281-286.

[11] Mbuntum, F.F. and Varadarajan, K., Half-exact pre-radicals. Comm. Alg. 5 (1977), 555-590.

[12] Ramamurthi, V.S., On splitting cotorsion radicals. Proc. Amer. Math. Soc. 39 (1973), 457-461.

[13] Robson, J. C., A note on Dedekind prime rings. Bull. London Math. Soc. 3 (1971), $42-46$.

[14] Rubin, R.A., Absolutely torsion-free rings. Pacific J. Math. 46 (1973), 503-514.

[15]][Rubin, R.A., Semi-simplicity relative to kernel functors. Canad. J. Math. 26 (1974), 1405-1411.

[16] Stenström, B., Rings of quotients. Die Grundlehren der mathematischen Wissenschaften in Einzeldarstellungen 217, Springer-Verlag, Berlin Heidelberg New York (1975).

[17] Viola-Prioli, J., On absolutely torsion-free rings. Pacific J. Math. 56 (1975), 275283.

Department of Mathematics

Yamaguchi University

Yamaguchi, 753 Japan 